\title{
Case Report: Parasitic Leiomyoma
}

\author{
G. Yared1,2, J. Darido1,2*, R. Chahine1, J. Khadra1,2, R. Bachnak¹,2, R. Bou Ghanem¹, K. Dimashkie', \\ M. Kharroubi ${ }^{1,2}$, I. Farhat ${ }^{3}$, I. Hatoum ${ }^{1}$
}

${ }^{1}$ Department of Obstetrics and Gynecology, Rafic Hariri University Hospital, Beirut, Lebanon

${ }^{2}$ Department of Obstetrics and Gynecology, The Lebanese University, Beirut, Lebanon

${ }^{3}$ Department of Urology Surgery, Rafic Hariri University Hospital, Beirut, Lebanon

Email: *jesydarido@hotmail.com

How to cite this paper: Yared, G., Darido, J., Chahine, R., Khadra, J., Bachnak, R., Ghanem, R.B., Dimashkie, K., Kharroubi, M., Farhat, I. and Hatoum, I. (2019) Case Report: Parasitic Leiomyoma. Journal of Cancer Therapy, 10, 722-726.

https://doi.org/10.4236/jct.2019.109060

Received: July 10, 2019

Accepted: August 30, 2019

Published: September 3, 2019

Copyright (C) 2019 by author(s) and Scientific Research Publishing Inc. This work is licensed under the Creative Commons Attribution International License (CC BY 4.0).

http://creativecommons.org/licenses/by/4.0/

\begin{abstract}
Parasitic Leiomyoma (PL) is considered a rare variant of uterine fibroids occurring outside the uterus. We hereby report a challenging case of parasitic leiomyoma that is considered rare in the literature with all its particularities. A 62 YO lady is known to have a history of diabetes, right breast carcinoma, appendectomy and normal vaginal deliveries presenting with a two-year history of back and abdominal pain with recent urinary symptoms. Therefore, an abdominopelvic $\mathrm{Ct}$ scan and MRI were done showing uterine fibroids compressing the bladder. The patient underwent an exploratory laparotomy. A palpable intravesical mass was identified. Transurethral cystoscopic resection was performed. Pathology results confirmed the leiomyomatous nature of the mass. Postoperatively, the surgery was complicated with a wound dehiscence and a hematoma formation on the left lateral wall of the bladder that was treated conservatively with a good follow-up outcome.
\end{abstract}

\section{Keywords}

Parasitic Leiomyoma, Uterine Fibroids

\section{Introduction}

Parasitic Leiomyoma (PL) is considered a rare variant of uterine fibroids occurring outside the uterus. In recent years, reported cases about PL have increased and their incidence is rising. Most of them occur in women of reproductive age. The formation of PL was attributed to a previous history of laparoscopic morcellation of fibroids (secondary PL). However, some patients diagnosed with PL, have no previous myomectomy or hysterectomy or any other uterine surgery (Primary PL) [1].

The management of PL is a true challenge [2]. Usually, the surgical resection 
of a PL is performed either open or laparoscopic or robotic. The most important issue is to perform a meticulous examination in order to assess the blood supply of the mass so the resection would be safe [3].

We hereby report a challenging case of parasitic leiomyoma that is considered rare in the literature with all its particularities so it will be an addition to the total of reported cases worldwide.

\section{Case Presentation}

This is a case of 62 YO non-smoker lady, G4P2A2, previous two normal vaginal deliveries and two abortions, known to have a history of diabetes and a right breast carcinoma treated on 2012 with a right mastectomy associated with chemotherapy, radiotherapy. She took six chemotherapy sessions, thirty radiotherapy sessions and she is currently on tamoxifen. On the other hand the patient has a past surgical history of appendectomy, tonsillectomy and pilonidal cyst excision.

She presented with back and abdominal pain at the emergency department of Rafic Hariri University Hospital. Her history goes back to two years ago when she started complaining about this pain but did not do any investigations about it. Then, she started having urinary symptoms (urgency, frequency, nocturia) on the three months that preceded her presentation. Therefore, an abdominopelvic Ct scan (Figure 1)/MRI (Figure 2) were done upon admission showing uterine fibroids compressing the bladder. An additional workup returned with normal left breast mammogram, Chest CT scan and Papsmear. CEA marker was 14.

The patient was admitted for total abdominal hysterectomy and bilateral salpingo ooporectomy via an exploratory laparotomy.

Under general anesthesia, through an infraumbilical midline incision, dissection layer by layer till reaching the peritoneum. Uterus and bilateral peritoneum are healthy. The bladder is visualized. A palpable intravesical mass was felt. Therefore, we have consulted the urology team. After inspection, they decided to proceed with cystoscopy and transurethral resection. The fascia, subcutaneous layer and skin were closed layer by layer. The patient was left intubated under general anesthesia and prepared for cystoscopy in lithotomy position. Upon inspection, there is a mass in the left lateral wall of the bladder just above the bladder neck. Urine cytology was taken and we proceeded for a transurethral resection of bladder tumor (TURBT) that was sent for pathology. Hemostasis was observed and reassured at the end of the procedure and a three-way foley was inserted.

The patient was put on amoxicillin as postoperative antibiprophylaxis, Low Molecular Weight Heparin for Deep Venous Thrombosis prophylaxis and incentive spirometer. At Day 1, she presented one episode of fever relieved with intravenous Paracetamol and was since then afebrile. At day 2, she passed flatus. At day 3, no fever, good ambulation, good bowel movement, foley was removed and at day 4 , she was discharged home. 


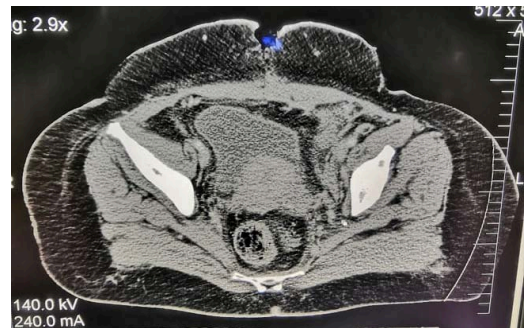

Figure 1. Ct scan showing uterine fibroid compressing the bladder.

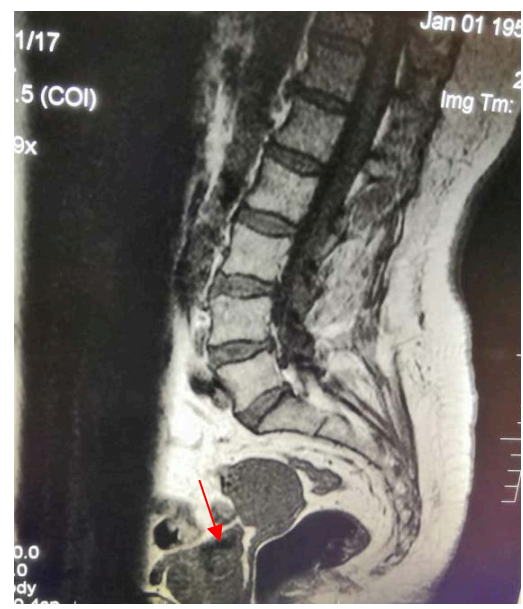

Figure 2. MRI showing uterine fibroids (arrow) compressing the bladder.

At day 7, the patient presented for fresh blood oozing at the incision site. An abdominopelvic $\mathrm{Ct}$ scan revealed wound dehiscence with subcutaneous fatty strandings and a tract through the rectus fascia suggesting facial dehiscence. A hematoma was also seen on the left lateral wall of the bladder measuring $8.2 \times$ $3.4 \mathrm{~cm}$. CRP $12 \mathrm{WBC}$ 6600. She was put on imipenem/cilastatin and treated conservatively with a good follow-up outcome.

Note that the pathology results turned back with a retroperitoneal Leiomyomatous mass weighing $47.4 \mathrm{~g}$ and measuring $6 \times 4 \times 3 \mathrm{~cm}$ adjacent to bladder neck with rare tiny calcifications.

The patient was lost to follow-up. Consequently, we have no information to be reported about her late stage.

\section{Discussion}

This is an unusual case of benign leiomyomatous tumor starting from the patient's background to the intraoperative diagnosis, management and postoperative follow-up.

From the four main types of uterine fibroids, parasitic Leiomyoma was considered by the authors Grover et al., and Shaudian et al. as a subtype of subserosal fibroid. [2] [4]. By the FIGO classification of uterine fibroids, PL is a type 8 leiomyoma with neither a myometrial involvement nor a uterine attachment [5]. 
On the other hand, unlike most of the reported cases in the literature [6] [7] [8], our case has no previous history of laparoscopic myomectomy that may provoke unintentional seeding of fragments. In other words, she has no evident predisposing iatrogenic factor for PL. So the etiology is unknown here. Note that appendectomy as an abdominal surgery cannot be considered as a significant indirect causative factor of PL.

As for the preoperative imaging, neither the abdominopelvic Ct scan nor abdominopelvic MRI gave us clear evidence for the diagnosis of PL.

On the other hand, the most common reported locations of PL are: the broad ligament, pelvic peritoneum, cul-de-sac and omentum [9]. However, in our case, the $\mathrm{PL}$ is located in the intravesical area. Consequently to this location, a transurethral cystoscopic complete resection was performed and this kind of management is not routine for uterine leiomyomas.

\section{Conclusion}

Finally, this case is rare in the literature. More cases of PL and uterine leiomyomas need to be reported and studies need to be made so we can, at first, specify modifiable risk factors in patients who have no previous uterine surgery predisposing them to such a mass and, secondly, develop the preoperative diagnostic methods including the imaging characteristics.

\section{Funding}

No source to be stated.

\section{Consent}

Written consent has been taken from the patient for publication of this report.

\section{Conflicts of Interest}

The authors declare no conflicts of interest regarding the publication of this paper.

\section{References}

[1] Lete, I., González, J., Ugarte, L., Barbadillo, N., Lapuente, O. and Álvarez-Sala, J. (2016) Parasitic Leiomyomas: A Systematic Review. The European Journal of $O b$ stetrics \& Gynecology and Reproductive Biology, 203, 250-259.

[2] Grover, A. and Bhalla, S. (2015) Parasitic Leiomyoma: A Rare Complication Following Laparoscopic Myomectomy with Review of Literature. Current Medicine Research and Practice, 5, 278-281. https://doi.org/10.1016/j.cmrp.2015.10.002

[3] Khan, A., Shawl, A. and Leung, P.S. (2018) Parasitic Leiomyoma of the Greater Omentum Presenting as Small Bowel Obstruction. Journal of Surgical Case Reports, 2018, rjy164. https://doi.org/10.1093/jscr/rjy164

[4] Schaudien, D., Muller, J.M. and Baumgartner, W. (2007) Omental Leiomyoma in a Male Adult Horse. Veterinary Pathology, 44, 722-726. https://doi.org/10.1354/vp.44-5-722 
[5] Kaplana, B. and Panda, S.R. (2018) Rare Presentation of a Case of Primary Parasitic Leiomyoma with Concurrent Uterine Leiomyomas. International Journal of Reproduction, Contraception, Obstetrics and Gynecology, 7, 2503-2505. https://doi.org/10.18203/2320-1770.ijrcog20182377

[6] Sarmalkar, M., Nayak, A., Singh, N., Mehendale, M. and Dixit, P. (2016) A Rare Case of Primary Parasitic Leiomyoma Mimicking as Ovarian Mass: A Clinical Dilemma. International Journal of Reproduction, Contraception, Obstetrics and Gynecology, 5, 545-548. https://doi.org/10.18203/2320-1770.ijrcog20160409

[7] Kimberley, A. and Nezhat, C. (2009) Parasitic Myomas. Obstetrics \& Gynecology, 114, 611-615. https://doi.org/10.1097/AOG.0b013e3181b2b09a

[8] Lu, B., Xu, J. and Pan, Z. (2016) Iatrogenic Parasitic Leiomyoma and Leiomyomatosis Peritonealis Disseminate Following Uterine Morcellation. Journal of Obstetrics and Gynaecology Research, 42, 990-999.

https://doi.org/10.1111/jog.13011

[9] Fasih, N., Shambogue, P., et al. (2008) Leiomyomas beyond the Uterus: Unusual Locations, Rare Manifestations. RadioGraphics, 28, 1931-1948.

https://doi.org/10.1148/rg.287085095 